sood reflection camera, which enabled a specimen to be set at suitable small angles of the order of a degree to the beam of electrons, be rotated in its own plane, also rotated so as to alter the angle of incidence, and be withdrawn and put back into place. This, with some modification, I believe has been the model for those that have followed.

\title{
Early Work on the Positron and Muon*
}

\author{
C. D. AndLRSON \\ California Institute of Technology, Pasadcna, California
}

(Received August 4, 1961)

\begin{abstract}
Early work on the positron and muon is described in an informal way, with emphasis on those aspects of the work which normally would not find their way into the literature.
\end{abstract}

T ODAY I plan to discuss briefly two discoveries with which I have had some association, the discovery of the positron and of the muon.

First, with respect to the positron, it has often been stated in the literature that the discovery of the positron was a consequence of its theoretical prediction by Dirac, but this is not true. The discovery of the positron was wholly accidental. Despite the fact that Dirac's relativistic theory of the electron was an adequate theory of the positron, and despite the fact that the existence of this theory was well known to nearly all physicists, it played no part whatsoever in the discovery of the positron.

The aim of the experiment that led to the discovery of the positron was simply to measure directly the energy spectrum of the secondary electrons produced in the atmosphere and other materials by the incoming cosmic radiation which at that time (1930) was thought to consist primarily of a beam of photons or gamma rays of energies of the order of several hundred millions of electron volts. Although there was no experimental evidence as to the detailed interactions between such a beam of high-energy photons and matter, it was presumed from experiments at lower energies that the dominant mechanism would be the production of highenergy secondary electrons by the Compton

* Paper delivered as part of a program on "Topics in the history of modern physics" on February 3, 1961, at a joint session of the American Physical Society and the American Association of Physics Teachers during their annual meetings in New York City. process, the discovery of which we have just heard discussed this afternoon by Professor Compton.

The apparatus employed was planned in 1930 by Professor Robert A. Millikan and myself and consisted of a cloud chamber operated in a strong magnetic field capable of producing measurable curvatures of electrons up to energies of a few billion electron volts.

The first result of the experiment was to show that the Compton process did not play an important role in the absorption of cosmic radiation, but that instead some new processes, presumably of a nuclear type, were operative. This was brought out by the fact that about half of the high-energy cosmic-ray particles observed were positively charged and therefore could not represent Compton electrons. At the time they were presumed to be protons resulting perhaps from photo-nuclear disintegrations. It was, of course, important to provide unambiguous identification of these unexpected particles of positive charge, and this could best be done by gathering whatever information was possible on the mass of the particles, inasmuch as the photographs clearly showed that in all cases these particles carried a single unit of electric charge. Experimental conditions were such that no information as to the particle's mass could be ascertained except in those cases in which the particle's velocity was appreciably smaller than the velocity of light, and this was true for only a small fraction of the events.

A few of the low-velocity particles were clearly 
identified as protons. As more data were accumulated, however, a situation began to develop which had its awkward aspects in that practically all of the low-velocity cases were particles whose mass seemed to be too small to permit their interpretation as protons. The alternative interpretations in these cases were that these particles were either electrons (of negative charge) moving upward or some unknown lightweight particles of positive charge moving downward. In the spirit of scientific conservatism we tended at first toward the former interpretation, i.e., that these particles were upward-moving negative electrons. This led to frequent and at times somewhat heated discussions between Professor Millikan and myself, in which he repeatedly pointed out that everyone knows that cosmic-ray particles travel downward, and not upward, except in extremely rare instances, and that therefore, these particles must be downwardmoving protons. This point of view was very difficult to accept, however, since in nearly all cases the specific ionization of these particles was too low for particles of proton mass.

To resolve this apparent paradox a lead plate was inserted across the center of the chamber in order to ascertain the direction in which these low-velocity particles were traveling and to distinguish between upward-moving negatives and downward-moving positives.

It was not long after the insertion of the plate that a fine example was obtained in which a lowenergy light-weight particle of positive charge was observed to traverse the plate, entering the chamber from below and moving upward through the lead plate. Ionization and curvature measurements clearly showed this particle to have a mass much smaller than that of a proton and, indeed, a mass entirely consistent with an electron mass. Curiously enough, despite the strong admonitions of Dr. Millikan that upward-moving cosmic-ray particles were rare, this indeed was an example of one of those very rare upward-moving cosmicray particles.

Soon additional cases of light-weight positive particles traversing the plate were observed, and in addition events in which several particles were simultaneously emitted from a common source were observed. Clearly in both types of cases the direction of motion was known, and it was therefore possible to identify the presence of several more light-weight positive particles whose mass was consistent with that of an electron but not with that of a proton.

After the existence of positrons was clearly indicated, the question naturally arose as to how they came into being. Just what was the mechanism responsible for their production? Naturally one would look to the Dirac theory to provide this explanation.

It was not immediately obvious to me, however, as to just what the detailed mechanism was. Did the positrons somehow acquire their positive charge from the nucleus? Could they be ejected from the nucleus when there were presumably no positrons present in the nucleus? The idea that they were created out of the radiation itself did not occur to me at that time, and it was not until several months later when Blackett and Occhialini suggested the pair-creation hypothesis that this seemed the obvious answer to the production of positrons in the cosmic radiation. Blackett and Occhialini suggested the pair-production hypothesis in their paper published in the spring of 1933, in which they reported their beautiful experiments on cosmic rays using the first cloud chamber which was controlled by Geiger counters.

Soon after this, experiments in which gamma rays were used showed that a pair of electrons, one positive and one negative, could be created in the coulomb field of a nucleus in such a way that the energy required to create the mass of the pair $2 m c^{2}$, and their kinetic energies as well, were supplied by the incident radiation, thus giving quantitative support to the pair-creation hypothesis.

The positron thus represents the first example of a particle consisting of antimatter. It is now generally believed that all particles have their corresponding antiparticles, and, in fact, several have recently been identified, including the antiproton and antineutron.

Progress on the theoretical side has continued, and a theory in the form of quantum-electrodynamics has been developed out of the Dirac theory by Feyman, Tomonaga, Schwinger, and others. It describes the interactions between charged particles and the electromagnetic field in a highly satisfactory manner. Also, calculations on the basis of the Dirac theory in terms of 
positron-electron pairs, which helped explain certain phenomena in the cosmic radiation such as the large cascade showers, were carried out by Oppenheimer, Carlson, Plesset, and others.

If one goes back a few years, say to just after the Dirac theory was announced, it is interesting then to speculate on what a sagacious per-. son working in this field might have done. Had he been working in any well equipped laboratory, and had he taken the Dirac theory at face value he could have discovered the positron in a single afternoon. The reason for this is that the Dirac theory could have provided an excellent guide as to just how to proceed to form positron-electron pairs out of a beam of gamma-ray photons. History did not proceed in such a direct and effcient manner, probably because the Dirac theory, in spite of its successes, carried with it so many novel and seemingly unphysical ideas, such as negative mass, negative energy, infinite charge density, etc. Its highly esoteric character was apparently not in tune with most of the scientific thinking of that day. Furthermore, positive electrons apparently were not needed to explain any other observations. Clearly the proton was the fundamental unit of positive charge, and the electron the corresponding unit of negative charge. This kind of thinking prevented most experimenters from accepting the Dirac theory wholeheartedly and relating it to the real physical world until after the existence of the positron was established on an experimental basis.

The discovery of the positron is also an example of a situation which is so often present in physics, in which the same discovery is made, or could easily have been made, in experiments simultaneously underway but carried out for quite different purposes. One such example is the famous experiment of Bothe and Becker in which a light nucleus such as Be was bombarded by $\alpha$ particles from a radioactive source. This experiment was first performed in 1930 by Bothe and Becker and later repeated by a number of investigators. As was shown later, this single simple experiment produced neutrons, positrons, and induced radioactivity. Had the positron not been discovered in cosmic-ray research, it would undoubtedly soon have been found in continuing studies of the experiment just described.

Concerning the muon, we know that the muon was the first particle to be discovered which has a mass between that of an electron and a proton. Originally it was known as the mesotron and later as the mumeson. More recent work on its properties has shown it is more appropriate not to classify it as a meson-type particle, and the suggestion has been made therefore to call it a muon. The term meson will be reserved for particles of intermediate mass which have a strong interaction with nuclei.

The muon, as I said, was the first particle of mass between a proton and an electron to be discovered. We now know, of course, from later experiments that a dozen or so such intermediate mass particles exist, including the pi mesons and a class of so-called strange particles called $k$ particles. I shall later speak briefly about these more recently discovered particles.

The discovery of the muon, unlike that of the positron, was not sudden and unexpected. Its discovery resulted from a two-year series of carefu1, systematic investigations all arranged to follow certain clues and to resolve some prominent paradoxes which were present in the cosmic rays.

The gist of the matter was as follows. Professor Seth H. Neddermeyer and I were continuing the study of cosmic-ray particles using the same magnet cloud chamber in which the positron was discovered. In these experiments it was found that most of the cosmic-ray particles at sea level were highly penetrating in the sense that they could transverse large thicknesses of heavy materials like lead and lose energy only by the directly produced ionization which amounted to something like 20 million ev per $\mathrm{cm}$ of lead. A principal aim of the experiments was to identify these penetrating cosmic-ray particles. They had unit electric charge and were therefore presumably either positive or negative electrons or protons, the only singly charged particles known at that time.

There were difficulties, however, with any interpretation in terms of known particles, as was pointed out as early as 1934 in a paper presented to the International Conference on Physics held in London of that year.

The most important objection to their interpretation as protons lay in the fact that the energy of the electron secondaries produced by the direct impact of these particles as observed 
in a cloud chamber contained too many secondaries of high energy to correspond with the energy spectrum to be expected if particles as massive as protons were producing these secondaries. On the other hand the spectrum was just that to be expected if the particles producing the secondaries were much lighter than protons. Furthermore, to interpret these particles as protons would mean assuming the existence of protons of negative charge since these sea-level particles occurred equally divided between negative and positive charges, and at that time there was no evidence for the existence of protons of negative charge.

There were difficulties also in interpreting these sea-level penetrating cosmic-ray particles as positive and negative electrons. The most important objections to their being electrons arose from three considerations. Firstly, theoretical calculations by Bethe, Heiter, and Sauter on the energy loss of electrons led to the conclusion that high-energy electrons should lose large amounts of energy through the production of radiation, which the penetrating particles in question were observed not to do. Secondly, we had found individual cases of electrons which did, in fact, show large energy losses through radiation, in some cases 100 million ev or more per $\mathrm{cm}$ of lead. Clearly in these cases the electrons showed a behavior quite different from that of the penetrating particles. And thirdly, the so-called highly absorbable component of the cosmic rays and the existence of electron showers could find an appealing explanation in terms of electrons if electrons did, in fact, suffer large radiation losses at high energies as demanded by the above mentioned theory.

This then was the situation in 1934 in which the sea-level penetrating particles had this paradoxical behavior. They seemed to be neither electrons nor protons. We tended, however, to lean toward their interpretation as electrons and "resolved" the paradox in our informal discussions by speaking of green electrons and red electrons-the green electrons being the penetrating type, and the red the absorbable type which lost large amounts of energy through the production of radiation.

Evidence of an entirely new type was soon obtained. In experiments carried out on the sum- mit of Pikes Peak in 1935 a number of cases of cosmic-ray produced nuclear disintegrations were observed from which many protons were ejected, but showing also in a few cases particles which, from ionization and curvature measurements, were lighter than protons and heavier than electrons. These observations were not conclusive evidence in themselves for the existence of a new type of particle, but they did tend to lend support to this assumption in view of the other difficulties involved in interpreting the data in terms of known particles.

The next year or so brought further evidence on all the above points and only tended to strengthen the paradox further. The hypothesis that the penetrating particles were protons was further weakened by the observation of many cases of particles which did not suffer appreciable radiation collisions and still which could not be as massive as protons, as evidenced by the ionization-curvature relations of their cloud-chamber tracks. These cases could, however, be interpreted as electrons, but only if electrons ceased to radiate appreciably above a certain energy, such as say a hundred million ev.

The crux of the matter then was whether or not electrons above a certain energy did or did not experience a large energy loss through radiative impacts. In other words the paradoxical character of our data could be reduced if one assumed that the Bethe-Heiter theory, although correct for electrons of energies below a few hundred million ev, in some way became invalid for electrons of higher energy, thus permitting high-energy electrons to have a much greater penetrating power and thus perhaps to permit interpreting the highly penetrating sea-level cosmic-ray particles as positive and negative electrons.

To test this hypothesis we inserted a bar of platinum across our cloud chamber and found that the cosmic-ray particles divided themselves into two groups, one highly absorbable and one highly penetrating in the same energy range. Thus the last possibility to explain the data in terms of known particles was removed, and it was necessary to assume the existence of positive and negative particles of unit charge but of a mass intermediate between that of a proton and 
an electron. These are the particles which are now called muons.

At about this time Street and Stevenson reported an experiment in which they observed particles which were lighter than protons and more penetrating than electrons should be if the Bethe-Heiter theory were valid. These they interpreted as particles of a new type, but this interpretation followed only if the Bethe-Heiter theory were assumed not to break down at the energies concerned in theit experiment.

In discussing the discovery of the muon I have not so far mentioned anything about the theoretical aspects of the situation. We saw previously how the Dirac theory predicted the existence of positrons although it played no role in their discovery. The discovery of muons similarly was based on purely experimental measurements and procedures, with no guide from any theoretical considerations.

As with the positron, this need not have been the case. For before the discovery of the muon had been finally achieved a novel idea was published in a Japanese journal by the Japanese physicist Hiedeka Yukawa. Reasoning by analogy with quantum clectrodynamics, he made the suggestion that perhaps nuclear forces (which are not electromagnetic in character) could be described in terms of a particle carrier of these nuclear forces, analogous to the photon being the carrier of electromagnetic forces. Nuclear forces, however, differ from electromagnetic forces in that they possess only a short range of action. This means that if nuclear forces are described in terms of a particle carrier, this particle carrier must have a finite rest mass unlike the photon of zero rest mass which is appropriate to the long range electromagnetic forces. Yukawa estimated from the known range of nuclear forces that this carrier should have a rest mass about 200 times that of an electron.

This novel suggestion of Yukawa was unknown to the workers engaged in the experiments on the muon until after the muon's existence was established. Although Yukawa's suggestion preceded the experimental discovery of the muon, he published it in a Japanese journal which did not have a general circulation in this country. It is interesting to speculate on just how much Yukawa's suggestion, had it been known, would have influenced the progress of the experimental work on the muon. My own opinion is that this influence would have been considerable even though Dirac's theory, which was much more specific than Yukawa's, did not have any effect on the positron's discovery. My reason for believing this is that for a period of almost two years there was strong and accumulating evidence for the muon's existence, and it was only the caution of the experimental workers that prevented an earlier announcement of its existence. I believe that a theoretical idea like Yukawa's would have appealed to the people carrying out the experiments and would have provided them with a belief that maybe after all there is some need for a particle as strange as a muon, especially if it could help explain something as interesting as the enigmatic nuclear forces. Yukawa's particle at that time did seem to coincide in all respects with the known properties of the muon.

And now having said this, my next statement will be that the muon and the Yukawa particle cannot possibly be the same particle at all. The Yukawa particle was invented to explain nuclear forces. The muon, as shown by subsequent experiments, completely ignores nuclear forces and interacts with other particles appreciably only through the electromagnetic field. It was not until 10 years later that another particle of intermediate mass, the pi meson, was discovered. The pi meson does interact strongly through nuclear forces and can indeed correspond to the Yukawa particle and is so considered in present day nuclear theory.

As we all know now, the muon is observed principally as a decay product of pi mesons. Muons may, however, also be produced in other ways, by direct pair production from high-energy photons and as decay products of many of the new so-called strange particles, the $k$ particles and hyperons.

The muon even today is somewhat of an oddball particle. Its existence does not in any way seem to be justified; for example, it seems to play no role whatever in explaining nuclear forces, whereas all the other more recently discovered intermediate mass particles do seem to be active participants as carriers of the nuclear forces.

It is interesting to contrast the spirit of the 
times when the positron and the muon were being discovered with the spirit of the times today. Today we include some 30 particles in our list of elementary particles, and new particles seem now to be discovered almost 2 or 3 at a time. This is certainly in sharp contrast with the reluctance and the conservatism which were present when particle numbers $3-5$ were being added to the list of elementary particles some years ago.

Although much new information about the properties of these 30 elementary particles is rapidly being acquired, there is much yet to be learned as far as an understanding of their role in nuclear physics is concerned, and as far as a satisfactory theoretical description of them is concerned.

Certain real progress, however, has been made in this direction through the interesting theoretical ideas of Pais, Gell-Mann, Feyman, Nishijima, Lee, Yang, and others. But a complete understanding will have to await new and better experiments and clearly new and more novel ideas.

Everyone has been hoping for and searching for a basically simple scheme of things, but this has not been achieved. Even the word "elementary" when" applied to the 30 particles loses its conventional meaning. Perhaps in the future a simplification will be reached. Most of us have faith that it will. For the time being, however, I think the situation is best summed up in terms of a modern definition of the word "elementary" which I believe is due to Robert Oppenheimer. An elementary particle is something so simple that one knows nothing whatsoever about it.

\title{
An Unusual Method of Solving the Harmonic-Oscillator Equation
}

\author{
Robert WeInStock \\ Department of Physics, Oberlin College, Oberlin, Ohio \\ (Received July 13, 1961)
}

The differential equation of the harmonic oscillator is solved by a method that employs techniques of the elementary calculus only.

\section{INTRODUCTION}

W

E consider the equation

$$
m \ddot{x}+r \dot{x}+k x=F_{0} \cos \omega t,
$$

where $m, r, k, F_{0}$, and $\omega$ are positive constants, and the superior dots indicate differentiation with respect to the time $t$. It is well known that (1) describes the motion of a damped harmonic oscillator subject to the influence of an externally applied sinusoidal force. ${ }^{1}$ It is the purpose of this paper to present a method of achieving the general solution of (1) without appealing to techniques employed in the usual systematic treatment of linear differential equations with constant coefficients; indeed one finds here the use of quite elementary calculus only, a fact that may be of practical utility for teachers of calcu-

\footnotetext{
1 Alternatively, Eq. (1) applies to the motion of charge in a circuit containing capacitance, inductance, and resistance in series with a source of sinusoidal emf.
}

lus-based introductory physics courses. Use of complex numbers is eschewed.

One step in our treatment of Eq. (1) is to show how its solution is reduced in a straightforward manner to the solution of the special case

$$
m \ddot{y}+r \dot{y}+k y=0 .
$$

Another step involves reducing the solution of Eq. (2) to that of

$$
m \ddot{z}+K z=0,
$$

with $K=k-\left(r^{2} / 4 m\right)>0 ;^{2}$ or with $K \leqslant 0,{ }^{3}$ to

$$
m \ddot{z}+(-4 m K)^{\frac{1}{2}} \dot{z}=0 .
$$

The only possibly new idea resides in the method of solving Eq. (3) carried out directly below in Sec. II.

\footnotetext{
2 "Underdamped."

" "Overdamped" if $K<0$, "critically damped" if $K=0$.
} 